\title{
Infinitely Many Eigenfunctions for Polynomial Problems: Exact Results
}

\author{
Yi-Chou Chen \\ Department of General Education, National Army Academy, Taoyuan 320, Taiwan \\ Correspondence should be addressed to Yi-Chou Chen; cycu.chou@gmail.com
}

Received 1 September 2014; Accepted 28 October 2014

Academic Editor: Gue Myung Lee

Copyright (C) 2015 Yi-Chou Chen. This is an open access article distributed under the Creative Commons Attribution License, which permits unrestricted use, distribution, and reproduction in any medium, provided the original work is properly cited.

Let $F(x, y)=a_{s}(x) y^{s}+a_{s-1}(x) y^{s-1}+\cdots+a_{0}(x)$ be a real-valued polynomial function in which the degree $s$ of $y$ in $F(x, y)$ is greater than or equal to 1 . For any polynomial $y(x)$, we assume that $T: \mathbb{R}[x] \rightarrow \mathbb{R}[x]$ is a nonlinear operator with $T(y(x))=F(x, y(x))$. In this paper, we will find an eigenfunction $y(x) \in \mathbb{R}[x]$ to satisfy the following equation: $F(x, y(x))=a y(x)$ for some eigenvalue $a \in \mathbb{R}$ and we call the problem $F(x, y(x))=a y(x)$ a fixed point like problem. If the number of all eigenfunctions in $F(x, y(x))=a y(x)$ is infinitely many, we prove that (i) any coefficients of $F(x, y), a_{s}(x), a_{s-1}(x), \ldots, a_{0}(x)$, are all constants in $\mathbb{R}$ and (ii) $y(x)$ is an eigenfunction in $F(x, y(x))=a y(x)$ if and only if $y(x) \in \mathbb{R}$.

\section{Introduction and Preliminaries}

Lenstra [1] investigated that

$$
F(x, y(x))=0
$$

in which $F(x, y)$ is a polynomial function over the algebraic rational number field $\mathbb{Q}(\alpha)$ (where $\alpha$ is an algebraic number). He found a polynomial $y=y(x) \in \mathbb{Q}(\alpha)[x]$ satisfying the polynomial equation

$$
F(x, y(x))=x
$$

Further, Tung [2] extended (2) to solve polynomial solutions (near solutions) $y(x) \in \mathbb{K}[x]$ ( $\mathbb{K}$ is a field) for the following equation:

$$
F(x, y(x))=a x^{m}
$$

where $a \in \mathbb{K}$ is a constant depending on the polynomial solution $y(x)$ and $m \in \mathbb{N}$ a given nonnegative integer.

Moreover, Lai and Chen [3-5] extended (3) to solve $y(x) \in \mathbb{R}[x]$ satisfying the polynomial equation as the form

$$
F(x, y(x))=a p^{m}(x), \quad x \in \mathbb{R}
$$

where $a \in \mathbb{R}, m \in \mathbb{N}, p(\cdot)$ is an irreducible polynomial in $x \in \mathbb{R}$, and the polynomial function $F(x, y): \mathbb{R} \times \mathbb{R} \rightarrow \mathbb{R}$ is written by

$$
F(x, y)=\sum_{i=0}^{s} a_{i}(x) y^{i} \quad \text { with } s \geq 1
$$

where $s$ denotes the degree $\operatorname{deg}_{y} F$ of $y$ in $F(x, y)$.

Recently, Chen and Lai $[6,7]$ research a quasicoincidence problem in which an arbitrary nonzero polynomial function $f(x) \in \mathbb{R}[x]$ is given as follows:

$$
F(x, y(x))=a f(x)
$$

where $a$ is a constant.

Definition 1 (Chen and Lai, [6]). A polynomial function $y=$ $y(x)$ satisfying (6) is called a quasicoincidence solution corresponding to some real number $a$. This number $a$ is called a quasicoincidence value corresponding to the polynomial solutions $y=y(x)$.

In this paper, we define a fixed point like problem in which the $y(x) \in \mathbb{R}[x]$ is replaced by the arbitrary polynomial 
$f(x) \in \mathbb{R}[x]$ throughout this paper. Then we restate (6) as the following equation:

$$
F(x, y(x))=a y(x)
$$

It is a new developed fixed point like problem. We call the polynomial (7) as a fixed point like problem. The number of all eigenfunctions in (7) may be infinitely many, or finitely many, or not solvable.

Since there may exist many eigenfunctions corresponding to the eigenvalue $a$, for convenience, we use the following notations to represent different situations:

(1) $\mathbf{E}_{\text {function }}$, the set of all eigenfunctions " $y(x)$ " satisfying (7);

(2) $\mathbf{E}_{\text {value }}$, the set of all eigenvalues " $a$ " satisfying (7);

(3) $\mathbf{E}_{\text {function }}(a)$, the set of all eigenfunctions " $y(x)$ " corresponding to a fixed eigenvalue " $a$ ".

For each $a \in \mathbb{R}$, the cardinal number of $\mathbf{E}_{\text {function }}(a)$, denoted by $\left|\mathbf{E}_{\text {function }}(a)\right|$, satisfies the following condition:

$$
\left|\mathbf{E}_{\text {function }}(a)\right| \leq \operatorname{deg}_{y} F(x, y) .
$$

In Section 2, we derive some properties of eigenfunctions of $F(x, y)$. If (7) has infinitely many eigenfunctions, the concerned properties are described in Section 3.

Throughout the paper, we denote the polynomial function by

$$
\begin{aligned}
F(x, y) & =a_{s}(x) y^{s}+a_{s-1}(x) y^{s-1}+\cdots+a_{1}(x) y+a_{0}(x) \\
& =\sum_{i=0}^{s} a_{i}(x) y^{i}
\end{aligned}
$$

with $s \geq 1$. Moreover, we may assume that $a_{0}(x)$ is nonzero. Since $a_{0}(x)=0$, problem (7) may become

$$
a_{s}(x) y^{s}(x)+a_{s-1}(x) y^{s-1}(x)+\cdots+a_{1}(x) y(x)=a y(x) .
$$

Moreover, if problem (7) has infinitely many eigenfunctions, dividing $y(x)$ by both sides of the above equation, then there may exist infinitely many nonzero eigenfunctions $y(x)$ satisfying

$$
a_{s}(x) y^{s-1}(x)+a_{s-1}(x) y^{s-2}(x)+\cdots+a_{1}(x)=a
$$

for some $a \in \mathbb{R}$. Therefore, this problem becomes a special case of (3).

\section{Some Lemmas and a Former Theorem}

Throughout this paper, we consider (7) for the polynomial function (9).

Lemma 2. Let $y(x) \in \mathbf{E}_{\text {function. Then }}$

$$
y(x)=d p(x) \text { for some } d \in \mathbb{R},
$$

and this $p(x)$ is divisible $a_{0}(x)$ and is denoted by $p(x) \mid a_{0}(x)$.
Proof. Since $y(x) \in \mathbf{E}_{\text {function, }}$, we have $F(x, y(x))=a y(x)$ for some $a \in \mathbb{R}$. This means

$$
\begin{aligned}
& a_{s}(x) y^{s}(x)+a_{s-1}(x) y^{s-1}(x)+\cdots+a_{1}(x) y(x)+a_{0}(x) \\
& =a y(x)
\end{aligned}
$$

for some $a \in \mathbb{R}$. It leads to

$$
\begin{aligned}
y(x) & \left(a_{s}(x) y^{s-1}(x)+a_{s-1}(x) y^{s-2}(x)+\cdots+\left(a_{1}(x)-a\right)\right) \\
& =-a_{0}(x)
\end{aligned}
$$

then $y(x)$ is a factor of $a_{0}(x)$.

In Lemma 2, any eigenfunction is a factor $p(x)$ of $a_{0}(x)$. Thus we define a class of this factor as follows.

Notation 1. Let $p(x) \in \mathbb{R}[x]$, and we denote $\Phi(p(x))=$ $\{\alpha p(x): \alpha \in \mathbb{R}\}$.

According to Notation 1 , it is obvious that for any $p(x)$ in $\mathbb{R}[x]$, we have the cardinal number

$$
|\Phi(p(x))|=\infty
$$

For convenience, we explain the relations of $\mathbf{E}_{\text {function }}$ and $\Phi(p(x))$ in the following lemma.

Lemma 3. Consider

$$
\mathbf{E}_{\text {function }}=\bigcup_{p(x) \mid a_{0}(x)} \Phi(p(x)) \cap \mathbf{E}_{\text {function }} .
$$

Proof. For any $y(x) \in \mathbf{E}_{\text {function }}$, by Lemma 2, we have $y(x)$ | $a_{0}(x)$. That is,

$$
y(x) \in \Phi(p(x))
$$

for some factor $p(x)$ of $a_{0}(x)$. It follows that

$$
\mathbf{E}_{\text {function }} \subseteq \bigcup_{p(x) \mid a_{0}(x)} \Phi(p(x))
$$

and we obtain

$$
\mathbf{E}_{\text {function }}=\bigcup_{p(x) \mid a_{0}(x)} \Phi(p(x)) \cap \mathbf{E}_{\text {function }} .
$$

We will use the definitions of "the pigeonhole principle," which concert with Grimaldi [8] and the above relation can be explained as the following lemma.

Lemma 4. Suppose that the cardinal number $\left|\mathbf{E}_{\text {function }}\right|=\infty$; there exists a factor $p(x)$ of $a_{0}(x)$ such that the cardinal number

$$
\left|\Phi(p(x)) \cap \mathbf{E}_{\text {function }}\right|=\infty \text {. }
$$


Proof. By Lemma 3, we obtain

$$
\begin{aligned}
(\infty=)\left|\mathbf{E}_{\text {function }}\right| & =\left|\bigcup_{p(x) \mid a_{0}(x)} \Phi(p(x)) \cap \mathbf{E}_{\text {function }}\right| \\
& \leq \sum_{p(x) \mid a_{0}(x)}\left|\Phi(p(x)) \cap \mathbf{E}_{\text {function }}\right| .
\end{aligned}
$$

Since the number of all factor $p(x)$ of $a_{0}(x)$ is at most $2^{\operatorname{deg} a_{0}(x)}$, by pigeonhole's principle, it yields

$$
\left|\Phi(p(x)) \cap \mathbf{E}_{\text {function }}\right|=\infty
$$

for some factor $p(x)$ of $a_{0}(x)$.

In order to solve the problem (7), [6, Lemma 3 and Theorem 11] are needed as follows.

Lemma 5 (see [6, Lemma 3]). Assume that the number of all quasicoincidence solutions (defined in Definition 1) is infinitely many; then, for any two quasicoincidence solutions $y_{1}(x)$ and $y_{2}(x)$, we have

$$
y_{1}(x)-y_{2}(x)=\lambda g(x)
$$

for some constant $\lambda \in \mathbb{R}$ and some factor $g(x)$ of $f(x)$.

Theorem 6 (see [6, Theorem 11]). Assume that the number of all quasicoincidence solutions (defined in Definition 1) is infinitely many; then

$$
F(x, y)=\sum_{i=0}^{s} c_{i} \frac{f(x)}{g^{i}(x)}\left(y-y_{1}(x)\right)^{i}
$$

for some $y_{1}(x) \in \mathbb{R}[x], g(x)$ is a factor of $f(x)$, and $c_{i} \in \mathbb{R}$ for $i=0,1, \ldots, s$.

\section{Main Theorems}

In this section, we consider $F(x, y)=a y(x)$ for the polynomial function $F(x, y)$ defined in (9).

We investigate the fixed point like problem of simple polynomial functions $F(x, y)$ with $s=1$ at first. Theorems 7 and 8 describe the necessary and sufficient results of these simple functions.

Theorem 7. Let $F(x, y)$ be a polynomial function with $\operatorname{deg}_{y} F=1$ as the form $F(x, y)=a_{1}(x) y+a_{0}(x)$ for some $a_{1}(x), a_{0}(x) \in \mathbb{R}[x]$. If the cardinal number $\left|\mathbf{E}_{\text {function }}\right|=\infty$, then

(i) $a_{1}(x) \in \mathbb{R}$;

(ii) any eigenfunction $y(x)$ of (7) is of the form

$$
y(x)=\lambda a_{0}(x)
$$

for some $\lambda \in \mathbb{R}$.
Proof. Since $\left|\mathrm{E}_{\text {function }}\right|=\infty$, by Lemma 4, there exists a factor $p(x)$ of $a_{0}(x)$ such that

$$
\left|\Phi(p(x)) \cap \mathbf{E}_{\text {function }}\right|=\infty .
$$

There exist two different eigenfunctions $y_{1}(x), y_{2}(x) \in$ $\Phi(p(x)) \cap \mathbf{E}_{\text {function }}$ with

$$
\begin{aligned}
& y_{1}(x)=\alpha_{1} p(x), \\
& y_{2}(x)=\alpha_{2} p(x),
\end{aligned}
$$

for different constants $\alpha_{1}, \alpha_{2} \in \mathbb{R}$. Since $y_{1}(x), y_{2}(x) \in$ $\mathbf{E}_{\text {function, }}$, we have

$$
\begin{aligned}
& F\left(x, y_{1}(x)\right)=a y_{1}(x), \\
& F\left(x, y_{2}(x)\right)=b y_{2}(x),
\end{aligned}
$$

where $a, b \in \mathbf{E}_{\text {value. }}$. It follows that

$$
\begin{aligned}
& F\left(x, y_{1}(x)\right)=a_{1}(x) y_{1}(x)+a_{0}(x)=a y_{1}(x) \\
& F\left(x, y_{2}(x)\right)=a_{1}(x) y_{2}(x)+a_{0}(x)=b y_{2}(x) .
\end{aligned}
$$

By (27) and (29), we have

$$
\begin{aligned}
& a_{1}(x)\left(\alpha_{1} p(x)\right)+a_{0}(x)=a\left(\alpha_{1} p(x)\right), \\
& a_{1}(x)\left(\alpha_{2} p(x)\right)+a_{0}(x)=b\left(\alpha_{2} p(x)\right) .
\end{aligned}
$$

By (30), we get

$$
a_{1}(x)\left(\alpha_{1} p(x)-\alpha_{2} p(x)\right)=a \alpha_{1} p(x)-b \alpha_{2} p(x) .
$$

Since $\alpha_{1} \neq \alpha_{2}$ and $p(x)$ is nonzero, it follows that

$$
a_{1}(x)=\frac{a \alpha_{1}-b \alpha_{2}}{\alpha_{1}-\alpha_{2}} \in \mathbb{R} .
$$

For any $y(x) \in \mathbf{E}_{\text {function, }}$, we have

$$
F(x, y(x))=a_{1}(x) y(x)+a_{0}(x)=a y(x) .
$$

By (32), we let $a_{1}(x)=\widetilde{a_{1}} \in \mathbb{R},(33)$ becomes

$$
\widetilde{a_{1}} y(x)+a_{0}(x)=a y(x),
$$

and it follows that

$$
a_{0}(x)=\left(a-\widetilde{a_{1}}\right) y(x) .
$$

Owing to $a_{0}(x) \neq 0$, then we obtain

$$
y(x)=\tilde{\lambda} a_{0}(x)
$$

where $\tilde{\lambda}=1 / a-\widetilde{a_{1}}$.

The following theorem is the sufficient conditions of Theorem 7.

Theorem 8. Let $F(x, y)$ be a polynomial function with $\operatorname{deg}_{y} F=1$ as the form $F(x, y)=a_{1}(x) y+a_{0}(x)$ for some $a_{1}(x)$, $a_{0}(x) \in \mathbb{R}[x]$. If 
(i) $a_{1}(x) \in \mathbb{R}$,

(ii) any eigenfunction $y(x)$ of (7) is of the form

$$
y(x)=\lambda a_{0}(x)
$$

for some $\lambda \in \mathbb{R}$,

then $\left|\mathbf{E}_{\text {function }}\right|=\infty$.

Proof. By (i), we let $a_{1}(x)=a_{1} \in \mathbb{R}$, then $F(x, y(x))=a y(x)$ for some $a \in \mathbb{R}$. This implies

$$
a_{1} y(x)+a_{0}(x)=a y(x)
$$

and then $y(x)=\left(1 /\left(a-a_{1}\right)\right) a_{0}(x)$ is an eigenfunction of $(7)$ for any constant $a \neq a_{1}$. It follows that

$$
\infty=\left|\left\{\frac{1}{a-a_{1}} a_{0}(x): a \in \mathbb{R}-\left\{a_{1}\right\}\right\}\right| \leq\left|\mathbf{E}_{\text {function }}\right| ;
$$

then $\left|\mathbf{E}_{\text {function }}\right|=\infty$.

In Theorems 7 and 8, problem (7) with $\operatorname{deg}_{y} F=1$ is introduced. In the following theorems, we deal with (7) with $\operatorname{deg}_{y} F \geq 2$ when the number of all eigenfunctions is infinitely many.

Theorem 9. Suppose that the cardinal number $\left|\mathbf{E}_{\mathrm{function}}\right|=\infty$ and $\operatorname{deg}_{y} F(x, y) \geq 2$. Then the polynomial $F(x, y)$ can be represented as

$$
F(x, y)=\sum_{i=0}^{s} \beta_{i} y^{i}
$$

for some constants $\beta_{i} \in \mathbb{R}$.

Proof. Since $\left|\mathrm{E}_{\text {function }}\right|=\infty$, by Lemma 4 , there exists a factor $p(x)$ of $a_{0}(x)$ satisfying

$$
\left|\Phi(p(x)) \cap \mathbf{E}_{\text {function }}\right|=\infty .
$$

Let $y_{1}(x)$ be an eigenfunction in $\Phi(p(x)) \cap \mathbf{E}_{\text {function }}$ such that

$$
F\left(x, y_{1}(x)\right)=a_{1} y_{1}(x)
$$

for some eigenvalue $a_{1} \in \mathbb{R}$. By Remainder Theorem, we get

$$
F(x, y)=\left(y-y_{1}(x)\right) F_{1}(x, y)+a_{1} y_{1}(x),
$$

where $F_{1}(x, y)$ is the quotient and $a_{1} y_{1}(x)$ is the remainder.

From the above identity and considering any eigenfunction $y(x)$ in $\Phi(p(x)) \cap \mathbf{E}_{\text {function }} /\left\{y_{1}(x)\right\}$ with $F(x, y(x))=$ ay $(x)$, we substitute (43) by taking $y=y(x)$ above and it becomes

$$
\begin{aligned}
& (\operatorname{ay}(x)=) F(x, y(x)) \\
& \quad=\left(y(x)-y_{1}(x)\right) F_{1}(x, y(x))+a_{1} y_{1}(x) .
\end{aligned}
$$

Since $y_{1}(x), y(x) \in \Phi(p(x))$, we have

$$
\begin{gathered}
y_{1}(x)=\lambda_{1} p(x), \\
y(x)=\lambda p(x)
\end{gathered}
$$

for some different constants $\lambda_{1}$ and $\lambda$. Substituting (45) and (46) into (44), it becomes

$$
a \lambda p(x)=\left(\lambda p(x)-\lambda_{1} p(x)\right) F_{1}(x, y(x))+a_{1} \lambda_{1} p(x)
$$

and it leads to

$$
F_{1}(x, y(x))=\frac{a \lambda-a_{1} \lambda_{1}}{\lambda-\lambda_{1}} \in \mathbb{R}
$$

for any eigenfunction $y(x) \in \Phi(p(x)) \cap \mathbf{E}_{\text {function }} /\left\{y_{1}(x)\right\}$.

By (48), there exist infinitely many quasicoincidence solutions in $\Phi(p(x)) \cap \mathbf{E}_{\text {function }} /\left\{y_{1}(x)\right\}$ to satisfy

$$
F_{1}(x, y)=a f(x)
$$

with $f(x)=1$. This problem is a quasicoincidence problem; then by Theorem 6 , we have

$$
F_{1}(x, y)=\sum_{i=0}^{s-1} c_{i} \frac{f(x)}{g^{i}(x)}\left(y-y_{1}(x)\right)^{i}
$$

Moreover, since $f(x)=1$ and $f(x) / g^{i}(x) \in \mathbb{R}[x]$ for any $i=0,1,2, \ldots, s-1$, it implies that $g(x) \in \mathbb{R}$ and by Lemma 5 , any $y_{2}(x), y_{3}(x) \in \Phi(p(x)) \cap \mathbf{E}_{\text {function }} /\left\{y_{1}(x)\right\}$, we have

$$
y_{2}(x)-y_{3}(x)=d g(x)=d^{\prime} \in \mathbb{R} .
$$

By definitions of $\Phi(p(x)), y_{2}(x)$, and $y_{3}(x)$ can also be represented as

$$
\begin{aligned}
& y_{2}(x)=\lambda_{2} p(x), \\
& y_{3}(x)=\lambda_{3} p(x)
\end{aligned}
$$

for some $\lambda_{2}, \lambda_{3} \in \mathbb{R}$. By (51), it follows that

$$
y_{2}(x)-y_{3}(x)=\left(\lambda_{2}-\lambda_{3}\right) p(x) \in \mathbb{R} .
$$

Moreover, by (53), this implies that $p(x) \in \mathbb{R}$ and by (45), $y_{1}(x) \in \mathbb{R}$, say, $y_{1}(x)=b_{1}$ and (50) implies that

$$
F_{1}(x, y)=\sum_{i=0}^{s-1} c_{i}\left(y-b_{1}\right)^{i}=\sum_{i=0}^{s-1} d_{i} y^{i}
$$

for some $d_{i} \in \mathbb{R}, i=0,1,2, \ldots, s-1$.

By (54), (43) implies that

$$
\begin{aligned}
F(x, y) & =\left(y-y_{1}(x)\right) F_{1}(x, y)+a_{1} y_{1}(x) \\
& =\left(y-b_{1}\right) \sum_{i=0}^{s-1} d_{i} y^{i}+a_{1} b_{1}=\sum_{i=0}^{s} \beta_{i} y^{i} .
\end{aligned}
$$

Conversely, if $F(x, y)$ can be expressed as in Theorem 9, then the cardinal number $\left|\mathbf{E}_{\text {function }}\right|=\infty$; this problem becomes the sufficient conditions of Theorem 9 . 
Theorem 10. Assume that

$$
F(x, y)=\sum_{i=0}^{s} \beta_{i} y^{i}
$$

for some $\beta_{i} \in \mathbb{R}$ for $i=0,1, \ldots, s$; then

$$
\left|\mathbf{E}_{\text {function }}\right|=\infty \text {. }
$$

Proof. For any $y(x)=c \in \mathbb{R}$,

$$
\begin{aligned}
F(x, y(x)) & =\sum_{i=0}^{s} c_{i} y^{i}(x) \\
& =\sum_{i=0}^{s} c_{i} c^{i} \text { (this is a constant) }=a c,
\end{aligned}
$$

for some $a=\sum_{i=0}^{s} c_{i} c^{i} / c \in \mathbb{R}$. Then $\mathbb{R} \subseteq \mathbf{E}_{\text {function }}$ and then $\left|\mathbf{E}_{\text {function }}\right|=\infty$.

In fact, if $\left|\mathbf{E}_{\text {function }}\right|=\infty$, then $\mathbf{E}_{\text {function }}=\mathbb{R}$ and we prove it as follows.

Theorem 11. If $\left|\mathbf{E}_{\text {function }}\right|=\infty$, we have

$$
\mathbf{E}_{\text {function }}=\mathbb{R} \text {. }
$$

Proof. Since $\left|\mathbf{E}_{\text {function }}\right|=\infty$, by the proof of Theorem 10, we have

$$
\mathbb{R} \subseteq \mathbf{E}_{\text {function }}
$$

Conversely, considering any $y(x) \in \mathbf{E}_{\text {function, we have }}$

$$
F(x, y(x))=\sum_{i=0}^{s} \beta_{i} y^{i}(x)=a y(x)
$$

for some eigenvalue $a \in \mathbb{R}$. By Lemma 2, we have $y(x) \mid \beta_{0}$; this implies $y(x) \in \mathbb{R}$ and then $\mathbf{E}_{\text {function }} \subseteq \mathbb{R}$. This proof is completed.

From Theorems 9 and 11, we can easily obtain the following two corollaries.

Corollary 12. Let $F(x, y)=\sum_{i=0}^{s} a_{i}(x) y^{i} \in \mathbb{R}[x, y], s \geq 2$, with $a_{j}(x) \notin \mathbb{R}$ for some $j$, then $\left|\mathbf{E}_{\text {function }}\right|<\infty$.

Proof. This result can be immediately obtained from Theorem 9.

Corollary 13. If there exists an eigenfunction $y(x) \in \mathbf{E}_{\text {function }}$ with $y(x) \notin \mathbb{R}$, then $\left|\mathbf{E}_{\text {function }}\right|<\infty$.

Proof. This result can be immediately obtained from Theorem 11.

From Theorems 7, 8, and 10 and Corollary 12, we provide some examples of fixed point like problem (7) for some $a \in \mathbb{R}$, which have infinitely many eigenfunctions and do not have infinitely many eigenfunctions as follows.
Example 14. In the following examples, we by the form of $F(x, y)$, we can decide whether the number of all eigenfunctions in (7) is infinitely many or not.

(1) If $(x, y)=x y+1$, there do not exist infinitely many eigenfunctions (Theorem 7).

(2) If $F(x, y)=x y+x$, there do not exist infinitely many eigenfunctions (Theorem 7).

(3) If $F(x, y)=y+x$, there exist infinitely many eigenfunctions and

$$
\mathbf{E}_{\text {function }}=\{\lambda x: \lambda \in \mathbb{R}\}(\text { Theorem } 8) .
$$

(4) If $F(x, y)=-y^{2}+7 y+1$, there exist infinitely many eigenfunctions and

$$
\mathbf{E}_{\text {function }}=\mathbb{R}(\text { Theorem 10). }
$$

(5) If $F(x, y)=x y^{2}+x y+1$, there do not exist infinitely many eigenfunctions (Corollary 12).

(6) If $F(x, y)=\sum_{i=0}^{s} c_{i} y^{i}+x, s \geq 2$, for any constants $c_{i} \in$ $\mathbb{R}$, there do not exist infinitely many eigenfunctions (Corollary 12).

We would like to provide one open problem as follows.

Further Development. For a real-valued polynomial function $F: \mathbb{R} \times \mathbb{R} \rightarrow \mathbb{R}$, if $\left|\mathrm{E}_{\text {function }}\right|<\infty$, can we find a coNP complete algorithm to solve all eigenfunctions $y=y(x)$ satisfying (7)?

\section{Conflict of Interests}

The author declares that there is no conflict of interests regarding the publication of this paper.

\section{Acknowledgment}

This research was partly supported by NSC 103-2115-M-539001, Taiwan.

\section{References}

[1] A. K. Lenstra, "Factoring multivariate polynomials over algebraic number fields," SIAM Journal on Computing, vol. 16, no. 3, pp. 591-598, 1987.

[2] S. P. Tung, "Near solutions of polynomial equations," Acta Arithmetica, vol. 123, no. 2, pp. 163-181, 2006.

[3] H.-C. Lai and Y.-C. Chen, "A quasi-fixed polynomial problem for a polynomial function," Journal of Nonlinear and Convex Analysis, vol. 11, no. 1, pp. 101-114, 2010.

[4] H. C. Lai and Y. C. Chen, "Quasi-fixed polynomial for vectorvalued polynomial functions on $\mathrm{R}^{n} \times \mathrm{R}$," Fixed Point Theory, vol. 12, no. 2, pp. 391-400, 2011.

[5] Y.-C. Chen and H.-C. Lai, "A non-NP-complete algorithm for a quasi-fixed polynomial problem," Abstract and Applied Analysis, vol. 2013, Article ID 893045, 10 pages, 2013. 
[6] Y.-C. Chen and H.-C. Lai, "New quasi-coincidence point polynomial problems," Journal of Applied Mathematics, vol. 2013, Article ID 959464, 8 pages, 2013.

[7] Y.-C. Chen, "Infinitely many quasi-coincidence point solutions of multivariate polynomial problems," Abstract and Applied Analysis, vol. 2013, Article ID 307913, 9 pages, 2013.

[8] R. P. Grimaldi, Discrete and Combinatorial Mathematics, 5th edition, 2003. 


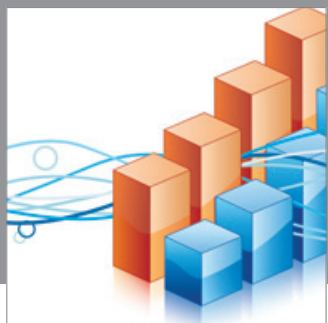

Advances in

Operations Research

mansans

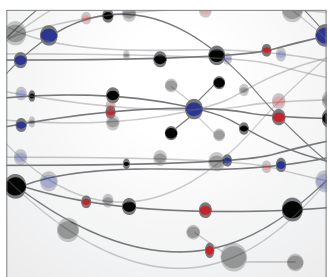

The Scientific World Journal
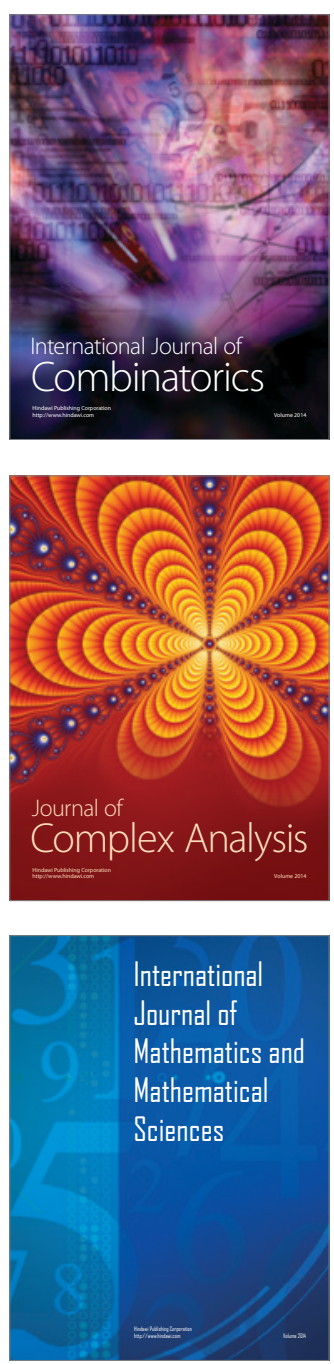
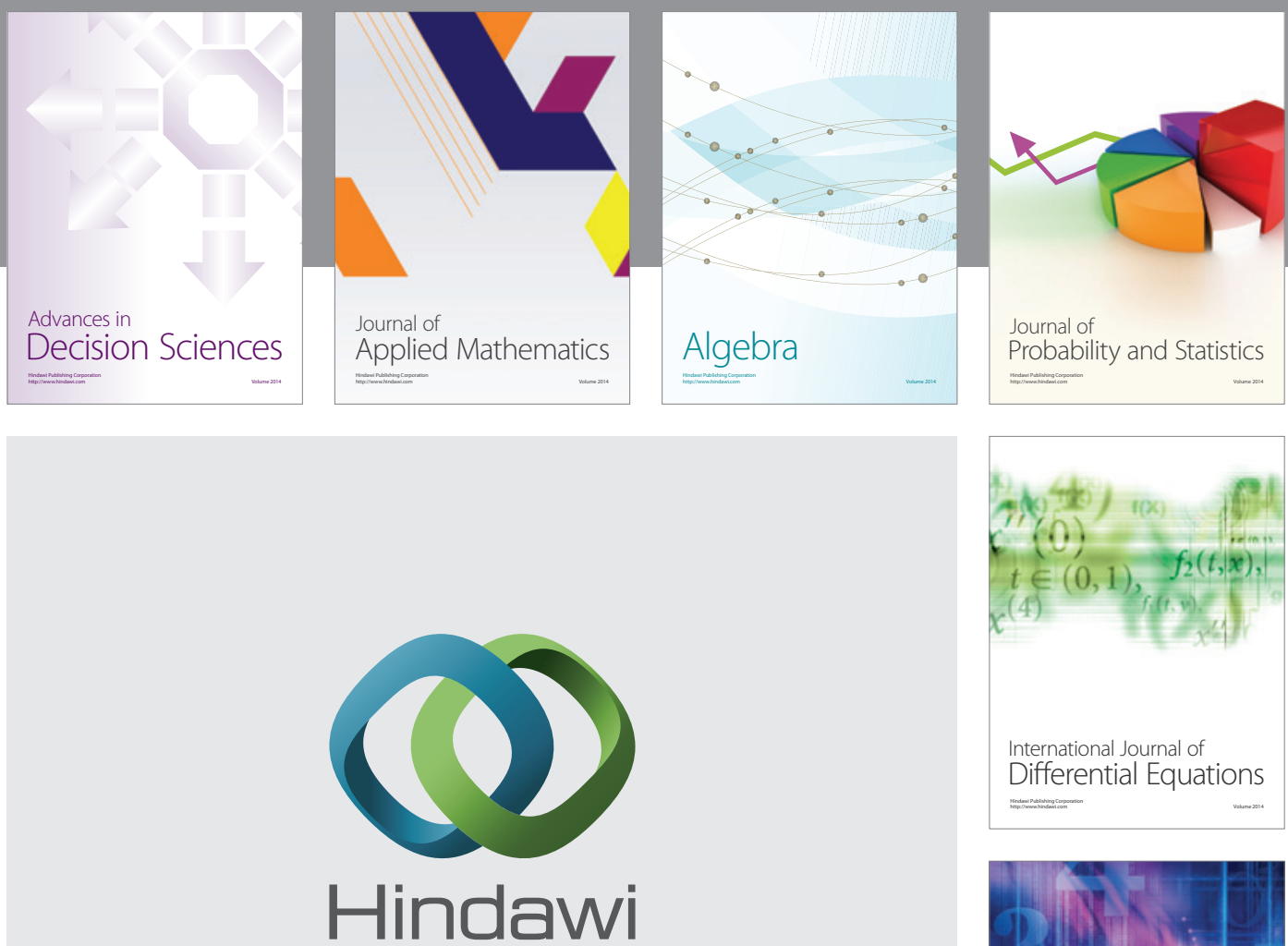

Submit your manuscripts at http://www.hindawi.com
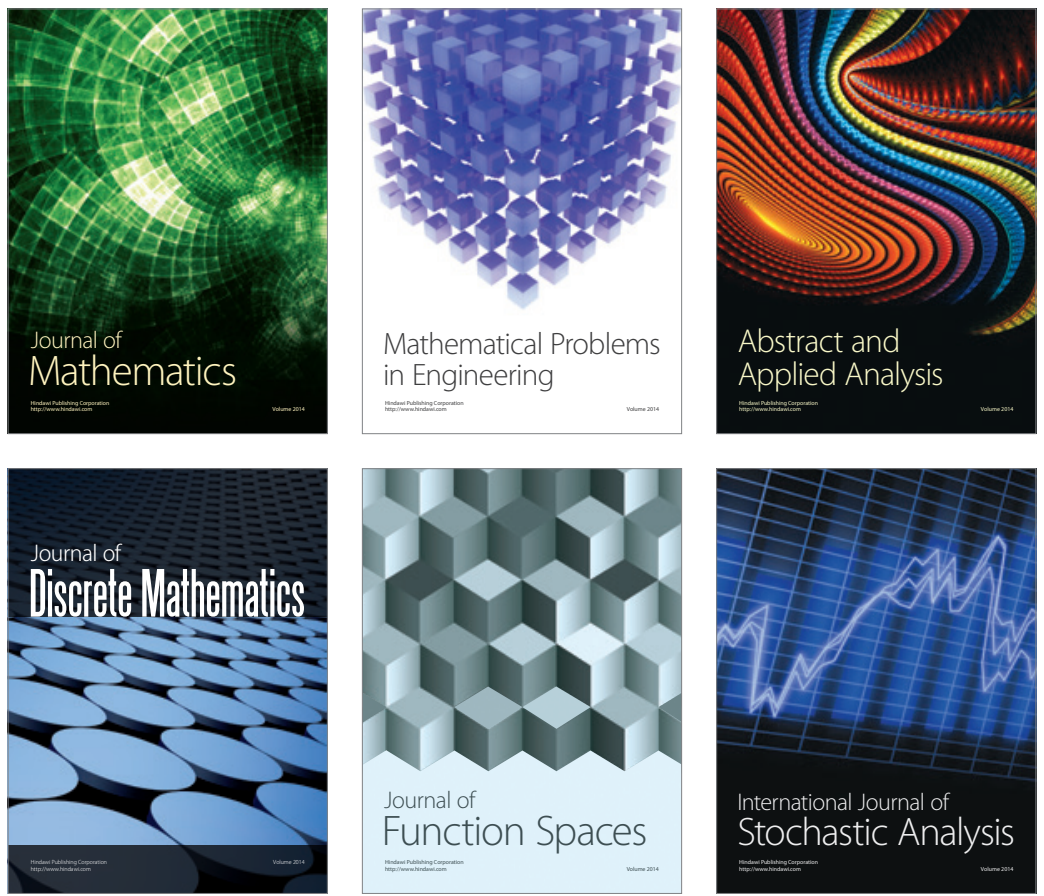

Journal of

Function Spaces

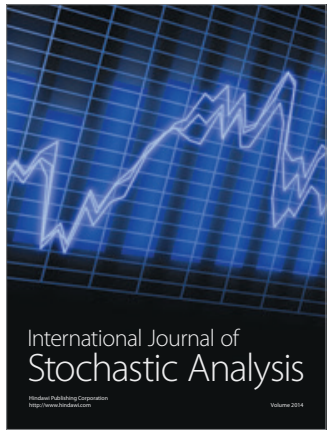

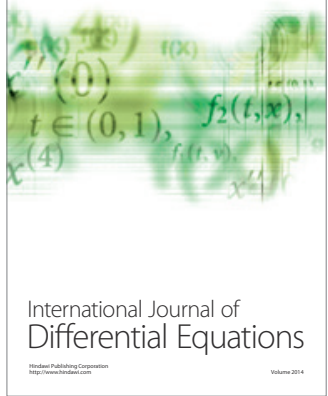
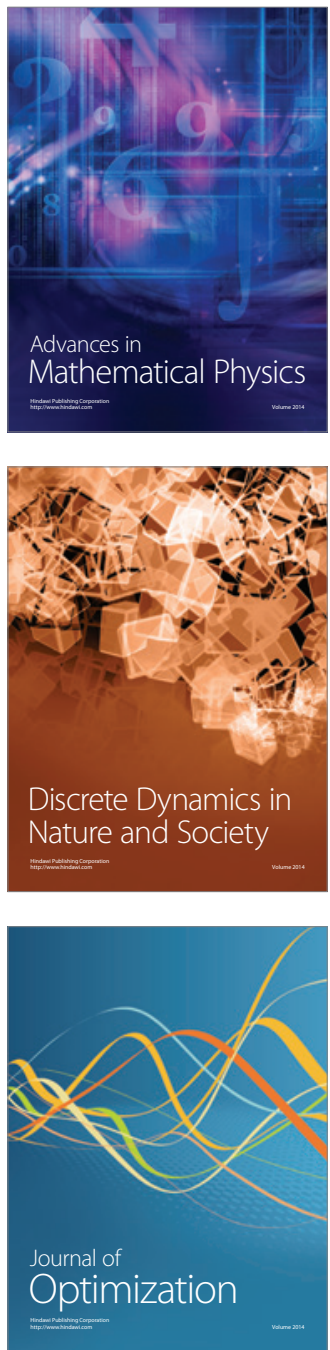